\title{
High prevalence of array comparative genomic hybridization abnormalities in adults with unexplained intellectual disability
}

\author{
Matthew R. G. Taylor, $M D, P h D^{I}$, Jean Jirikowic, $M S^{I}$, Cara Wells, $M S^{l}$, Michelle Springer, $M S^{2}$, \\ Loris McGavran, PhD ${ }^{2}$, Brenda Lunt, BS ${ }^{2}$, and Karen Swisshelm, $P h D^{2}$
}

\begin{abstract}
Purpose: Array comparative genomic hybridization is now a widely used clinical tool for the evaluation of intellectual disability. The current $10 \%$ yield of positive findings is based largely on pediatric data. Adults with unexplained intellectual disability have not been systematically studied with array comparative genomic hybridization. Here, we report our initial experience with array comparative genomic hybridization testing on 45 adults with unexplained intellectual disability referred to an adult genetics clinic. Methods: Beginning in 2006, we applied clinically available array comparative genomic hybridization testing to adults referred with an intellectual disability phenotype. The initial platform used was an early generation targeted or constitutional array, which was replaced by our current platform using more than 5000 bacterial artificial chromosome clones with an average resolution of 500 $\mathrm{Kb}$ and targeting 114 disease loci. All patients also underwent highresolution karyotype analysis and molecular testing for Fragile X syndrome. Results: Our population comprised 45 patients with unexplained intellectual disability (18 men and 27 women) with an average age of 35.1 years. Most patients had not been evaluated by genetics clinics since childhood or had never undergone a genetic evaluation; only two had documentation of prior normal karyotype studies. Three subjects had abnormal high-resolution chromosome studies, which were also confirmed by array comparative genomic hybridization. Seven of the remaining 42 patients (17\%) had novel genomic losses identified only by array comparative genomic hybridization. Conclusion: Abnormal genomic losses detected by array comparative genomic hybridization are prevalent in adults with unexplained intellectual disability. Our data showing abnormalities in $22 \%$ and $17 \%$ of overall patients and of cases with normal karyotypes, respectively, suggest that the yield of array comparative genomic hybridization in adults with unexplained intellectual disability may be higher than in pediatric populations. Genet Med 2010:12(1):32-38.
\end{abstract}

Key Words: intellectual disability, mental retardation, array comparative genomic hybridization, adult genetic disorders

ntellectual disability, formerly referred to as "mental retardation," is a serious birth defect of impaired cognitive and adaptive function with an onset of findings before 18 years of age. It is a common condition, prevalent in $1-3 \%$ of individuals, affecting $10 \%$ of families, and is an important public health

From the ${ }^{1}$ Department of Medicine, Adult Medical Genetics Program, and ${ }^{2}$ Department of Pathology, Colorado Genetics Laboratory, University of Colorado Denver, Denver, Colorado.

Matthew R. G. Taylor, MD, PhD, Mailstop F442, 12700 East 19th Avenue, Aurora, CO 80045. E-mail: matthew.taylor@ucdenver.edu.

Disclosure: The authors declare no conflict of interest.

Submitted for publication August 20, 2009.

Accepted for publication October 23, 2009.

Published online ahead of print December 18, 2009

DOI: 10.1097/GIM.0b013e3181c83de0 problem and societal cost in developed and underdeveloped countries. ${ }^{1-3}$ Genetic factors are suspected in a majority of cases, especially when obvious environmental insults have been excluded. The diagnostic yield of chromosome analysis and Fragile $\mathrm{X}$ testing depends on the population studied but are estimated to be abnormal in $5-10 \%$ and $2 \%$ of cases, respectively. ${ }^{4-6}$ In general, intellectual disability is suspected and confirmed in early childhood, and this is reflected in the literature where the majority of studies have been performed among pediatric cohorts.

The severity of intellectual disability correlates also with the yield of abnormal chromosomal findings, and the lowest prevalence of abnormalities are typically found in mild cases. Mild intellectual disability accounts for approximately $80 \%$ of cases and is comprised of individuals who often will engage in mainstream education programs, obtain employment, and enter into interpersonal relationships. Although survival among individuals with intellectual disability is reduced, those with a mild phenotype are most likely to survive into adulthood where they generally experience more health problems, hospitalizations, and account for more health care costs than the cognitively normal adult population. ${ }^{7-10}$ With improved pediatric care, the population of adults with intellectual disability will expand. Despite this growth, a majority of adults with this phenotype still lack a diagnosis for their condition, and many have not been evaluated by genetics since childhood. The lack of knowledge of an underlying diagnosis may limit the value of medical management and genetic counseling that can be applied to the patient and family.

Recently, the development of array comparative genomic hybridization $(\mathrm{aCGH})$ has transformed the evaluation of unexplained intellectual disability by uncovering a wide range of submicroscopic genomic deletions and gains in this population. The diagnostic yield of this technology depends again not only on the population studied but also on the aCGH platforms used, which differ among laboratories and continue to evolve. A recent meta-analysis of 19 large, well-designed studies of intellectual disability and congenital anomalies reported a clinically significant range of $6-35 \%$ for genomic imbalance and an overall diagnostic yield estimate of $10 \% .^{11}$ This study included data from 13,926 patients (range 20-8789/study), and although detailed age data are not reported in all studies, the studied population includes large populations from referral laboratories where sample ascertainment frequently comes from pediatricians and pediatric geneticists. One study of more than 5000 cases had a median age of just 4 years. ${ }^{12}$ Large laboratory-based studies may be protected to some ascertainment biases such as a focus on only severe cases but often experience difficulty obtaining complete clinical phenotype data. Some studies include aCGH data from children with dysmorphic features and congenital anomalies where a majority are $<2$ years of age, before cognitive phenotypes can often be clearly established. ${ }^{13}$ Specific data on adult populations, which may differ from 
children in terms of the prevalence and distribution of genomic gains and losses, have not been reported. Such data are needed to determine the benefit of aCGH and guide genetic counseling for these adults, many of who are of reproductive age.

\section{MATERIALS AND METHODS}

Patients were seen at the Adult Medical Genetics Clinic at the University of Colorado Hospital. The clinic is a referral center that broadly provides services for adults with known and suspected genetic conditions. Patients are referred by primary and subspecialty physicians for a diagnostic evaluation of their cognitive disability for the purpose of obtaining a specific diagnosis and affecting the counseling and management of patients and their families. All patients are seen by a single physician with board certifications in clinical genetics and in internal medicine and by a genetic counselor. Medical history and medical records are reviewed, and all subjects undergo high-resolution chromosomal analysis and fragile $\mathrm{X}$ testing. The severity of intellectual disability for each patient is based on intelligence quotient scoring, when available, and on the geneticist's clinical assessment based on other cognitive history and physical examination data. The minority of patients who presented to the clinic with a documented genetic explanation of their intellectual disability (i.e., prior abnormal karyotype) were excluded from this analysis. The study was reviewed and approved by our research institutional review board.

Clinical aCGH testing was performed by the Colorado Genetics Laboratory (Denver, CO). During the course of this study, two different aCGH platforms were sequentially used, reflecting the continued evolution of this technology: Spectral Genomics/Perkin Elmer Constitutional Chip ${ }^{\mathrm{TM}}$ v. 3.0 (Perkin Elmer, Waltham, MA) and BlueGnome CytoChip ${ }^{\mathrm{TM}}$ Versions 2.0/2.01, 3.0, and 3.01 (BlueGnome Limited ${ }^{\mathrm{TM}}$, Cambridge, UK). DNA was extracted from a peripheral blood sample using Gentra Puregene Blood kit from Qiagen (Valencia, CA) following the protocol for DNA purification from whole blood. Two separate array platforms were used depending on the sample acquisition date.

Samples from August 2006 to November $2007(N=11)$ were tested using arrays provided by Spectral Genomics ${ }^{\mathrm{TM}}$. Briefly, $2 \mu \mathrm{g}$ of each DNA was sonicated with a Sonic, Model VC-130 sonicator. DNA was purified with the use of the Zymo Clean-up Kit (Zymo Research, Orange, CA), and each DNA was separately labeled with cyanine-5 (Cy5) and cyanine-3 (Cy3) deoxycytidine triphosphates (dCTPs) using a labeling kit provided by the manufacturer (Spectral Genomics, Inc.). Labeled test and reference DNAs (forward hybridization: Cy3labeled test and Cy5-labeled reference; reverse hybridization: Cy5-labeled test and Cy3-labeled reference) were mixed, coprecipitated with isopropanol, washed, and resuspended in hybridization solution (Spectral Genomics). DNA mixtures were denatured at $72^{\circ} \mathrm{C}$ for 10 minutes, prehybridized at $37^{\circ} \mathrm{C}$ for 30 minutes, and cohybridized to the arrays for 16 hours at $37^{\circ} \mathrm{C}$ according to the manufacturer's recommended protocol. Forward and reverse hybridizations were performed using a constitutional array (Spectral Genomics/PerkinElmer Constitutional Chip ${ }^{\mathrm{TM}}$ v.3.0) consisting of 604 bacterial artificial chromosome (BAC) clones for 11 specimens. All clones were represented on the respective array in duplicate, with the genomic location of the clones provided by Spectral Genomics. After hybridization, the arrays were washed in 50\% formamide/ $2 \times$ saline sodium citrate (SSC), $2 \times \mathrm{SSC} / 0.1 \%$ Igepal, and $0.2 \times \mathrm{SSC}$ according to the manufacturer's recommendations, rinsed in Milli-Q water, dehydrated 5 minutes in 100\% ethanol, and immediately dried with compressed nitrogen gas. Images and signal intensities were acquired using a GenePix4000B (Axon Instruments, Burlingame, CA) dual-laser scanner in combination with GenePixPro 3.0 (Axon Instruments) imaging software.

Analysis of samples received after November $2007(N=34)$ were performed using the Cytochip v2.0/2.01, v3.0, or v3.01$0.5 \mathrm{Mb}$ Roswell Park BAC microarray platforms (BlueGnome Ltd., UK). Labeling reagents were obtained from BlueGnome Ltd. TM (Cytochip Consumable Pack-1-4131). The test and reference DNA were again labeled by random priming with Cy5$\mathrm{dCTP}$ and Cy3-dCTP to allow for forward and reverse analysis. The reaction was incubated overnight at $37^{\circ} \mathrm{C}$. Labeled test and control DNA was mixed with $25 \mu \mathrm{g}$ of Cot-DNA (Invitrogen, Carlsbad, CA) and $30 \mu \mathrm{g}$ of herring sperm and hybridized for 21 hours at $37^{\circ} \mathrm{C}$. Slides were washed two times in $2 \times$ $\mathrm{SSC} / 0.5 \%$ Igepal at room temperature for 10 minutes, $2 \times$ $\mathrm{SSC} / 0.5 \%$ Igepal at $60^{\circ} \mathrm{C}$ for 5 minutes, $1 \times \mathrm{SSC}$ at $60^{\circ} \mathrm{C}$ for 5 minutes, $0.1 \times \mathrm{SSC}$ at $60^{\circ} \mathrm{C}$ for 5 minutes, followed by $0.1 \times$ $\mathrm{SSC}$ wash for 5 minutes at room temperature. Slides were rinsed in Milli-Q water, dehydrated 5 minutes in \%100 ethanol, and immediately dried with compressed nitrogen gas. Arrays were scanned using GenePix ${ }^{\mathrm{TM}}$ 4000B scanner (Axon Instruments Inc., Union City, CA) and analyzed using BlueFuse (v3.5) imaging software (BlueGnome Ltd. ${ }^{\mathrm{TM}}$, Cambridge, UK).

\section{RESULTS}

Demographic characteristics of the first 45 consecutive adults with undiagnosed cognitive disability referred to the Adult Medical Genetics Clinic are presented in Table 1. The average age in this patient population was 35.1 years, and more women than men with cognitive disability were seen. The majority of

Table 1 Patient demographics for referrals for undiagnosed mental retardation from 2006 to 2009

\begin{tabular}{lcc}
\hline & Study cohort & Abnormal aCGH \\
\hline Total patients & 45 & 10 \\
Age $(\mathrm{yr})$ & $35.1(\mathrm{SD}=6)$ & $33.9(\mathrm{SD}=2)$
\end{tabular}

Gender (\%)

Male

$18(40)$

Female

$27(60)$

Race/ethnicity (\%)

Non-Hispanic White

34 (76)

Hispanic

$6(13)$

Black

$4(9)$

Other

$1(2)$

Average full-scale $\mathrm{IQ}^{a}$

$60.9(\mathrm{SD}=22.5)$

$54.2(\mathrm{SD}=15)$

Clinical degree of intellectual disability $(\%)$

$\begin{array}{lll}\text { Mild } & 19(42) & 3(30) \\ \text { Moderate } & 15(33) & 4(40) \\ \text { Severe/profound } & 11(24) & 3(30)\end{array}$

${ }^{a}$ When available. 
patients $(n=19)$ had mild intellectual disability and a prior negative genetic workup, including chromosome analysis, was documented in only 2 of the 45 cases before we initiated testing. Most of our patients had little to no contact with biological relatives, limiting our ability to obtain and independently confirm early developmental, medical, and/or family history data. Most patients were living in a supervised setting (family home, group home, and adult foster care) and participated in day programs through local community services. Approximately half of these individuals worked or volunteered on a part-time basis. Family history data were available in about one-half of visits, although the amount of family history data were often minimal and could not be independently confirmed.

Pathogenic aCGH findings were present in 10 of $45(22 \%)$ patients (Fig. 1 and Tables 2 and 3) including nine deletions and one patient having both a gain and a deletion of chromosomal material. In all cases, abnormal array results were confirmed by molecular cytogenetic (fluorescence in situ hybridization) studies or standard cytogenetic studies, depending on the size of the abnormality. These losses are presumed novel and pathogenic, being absent in known databases as genomic variants. The estimated minimum size of deletions ranged from $77.3 \mathrm{~Kb}$ to
9.7 Mb. One patient had both a genomic gain (8p23.3-8p21.3; $20.65 \mathrm{Mb})$ and a loss $(5 \mathrm{p} 15.33-5 \mathrm{p} 15.31 ; 9.6 \mathrm{Mb})$ as the result of a derivative chromosome. Initial standard cytogenetic studies had detected a 46,XX,add(5)(p15.1) karyotype. The aCGH results characterized this abnormality as being a derivative Chromosome 5 resulting from a translocation. In the cases of the two remaining patients with abnormal chromosome studies (Patients 2 and 5), the cytogenetic abnormalities were better defined by the aCGH. For Patient 2, a previous standard cytogenetic study reported a 46,XY, $\operatorname{del}(2)(\mathrm{q} 35 \mathrm{q} 37.1)$ karyotype. The aCGH results for this patient characterized the abnormality to be limited to $2 \mathrm{q} 36$. For Patient 5, initial cytogenetic studies revealed an apparently unbalanced abnormality within 5q14.2-q15. The aCGH clarified this abnormality to be 5q14.3-q15. Parental DNA samples were not available for study, with the exception of Patients 3 and 9. For Patient 3, a maternal fluorescence in situ hybridization study was found to be negative with a paternal sample being unavailable, and for Patient 9, both parents were tested, and the deletion was found to be de novo.

Four genomic gains were also identified (Tables 2 and 3; Patients 11-14). Three of these were initially suspected to be novel and potentially pathogenic; the fourth gain (Patient 13;

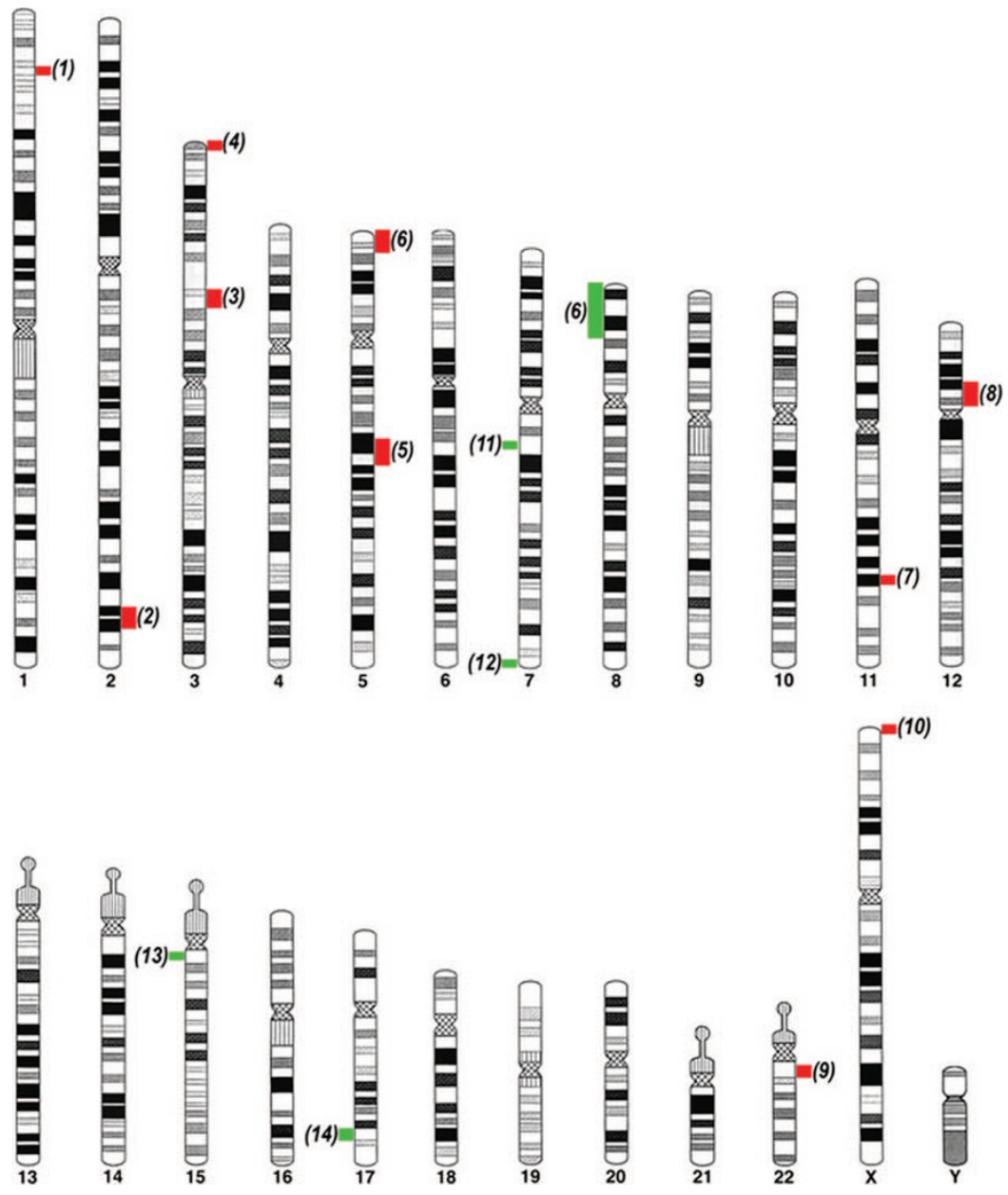

Fig. 1. Chromosome representation of the aCGH-detected genomic losses and gains in this study. Pathogenic genomic losses are represented by red blocks; genomic gains are represented by green blocks. The numbers in parentheses correspond to patient numbers as presented in Table 2. The figure is based on the International System for Human Cytogenetic Nomenclature. ${ }^{19}$ 
Table 2 Abnormal aCGH findings

\begin{tabular}{|c|c|c|c|c|c|c|c|c|}
\hline Patients & $\begin{array}{l}\text { aCGH } \\
\text { version }\end{array}$ & Gain/loss & $\begin{array}{l}\text { Chromosome } \\
\text { locus }\end{array}$ & $\begin{array}{l}\text { Linear } \\
\text { position } \\
(\mathrm{Mb})\end{array}$ & $\mathrm{PFC}(\mathrm{Mb})$ & $\begin{array}{l}\mathrm{DFC} \\
(\mathrm{Mb})\end{array}$ & $\begin{array}{l}\text { Minimum } \\
\text { size }\end{array}$ & Genes in region \\
\hline 1 & $\mathrm{CC} 3.0$ & Loss & $1 \mathrm{p} 36.11$ & 27.6 & 28.8 & 27.2 & $77.3 \mathrm{~Kb}$ & CD164L2, GPR3, WASF2 \\
\hline 2 & $\mathrm{CC} 3.0$ & Loss & $2 \mathrm{q} 36.1-2 \mathrm{q} 36.3$ & $222.6-230.5$ & 221.4 & 231.1 & $7.9 \mathrm{Mb}$ & $\begin{array}{l}\text { PAX3, COL4A4, COL4A3, } \\
\quad \text { SLC19A3 }\end{array}$ \\
\hline 3 & $\mathrm{CC} 2.0$ & Loss & $3 \mathrm{p} 21.1-3 \mathrm{p} 21.2$ & $51.47-52.77$ & 52.64 & 44.65 & $1.3 \mathrm{Mb}$ & ARMET, ACY1, TNNC1, DOCK3 \\
\hline 4 & $\mathrm{CC} 2.01$ & Loss & $3 \mathrm{p} 26.3$ & $0.23-1.03$ & 2.27 & 0.13 & $800 \mathrm{~Kb}$ & CNTN6 \\
\hline 5 & $\mathrm{CC} 3.0$ & Loss & $5 q 14.3-5 q 15$ & $87.3-93.6$ & 86.4 & 94.6 & $6.3 \mathrm{Mb}$ & GPR98, MEF2C \\
\hline \multirow[t]{2}{*}{6} & $\mathrm{CC} 3.0$ & Loss & $5 \mathrm{p} 15.33-5 \mathrm{p} 15.31$ & $0.23-9.79$ & 10.73 & Telomere & $9.56 \mathrm{Mb}$ & $\begin{array}{l}\text { SDHA, TERT, SLC6A19, } \\
\text { NDUFS6, MTRR }\end{array}$ \\
\hline & & Gain & $8 \mathrm{p} 23.3-8 \mathrm{p} 21.3$ & $0.15-20.8$ & 21.79 & Telomere & $20.65 \mathrm{Mb}$ & $\begin{array}{l}\text { MCPH1, GATA4, TUSC3, DLC1, } \\
\text { MFHAS1, ARHGEF10 }\end{array}$ \\
\hline 7 & $\mathrm{CC} 3.01$ & Loss & $11 \mathrm{q} 22.3$ & $103.9 \mathrm{Mb}$ & 103.0 & 104.7 & $161.5 \mathrm{~Kb}$ & CASP1, CASP4, CASP5, CASP12 \\
\hline 8 & $\mathrm{CC} 3.0$ & Loss & $12 \mathrm{p} 11.22-12 \mathrm{p} 12.1$ & $20.8-30.5$ & 30.8 & 20.1 & $9.7 \mathrm{Mb}$ & $\begin{array}{c}A B C C 9, \text { KRAS, GYS2, LDHB, } \\
\text { KCNJ8, PTHLH, BCAT1 }\end{array}$ \\
\hline 9 & $\mathrm{SGC}$ & Loss & $22 \mathrm{q} 11.2$ & $17.75-19.9$ & 17.33 & 23.59 & $2.15 \mathrm{Mb}$ & TBX1, SERPIND1, SNAP29 \\
\hline 10 & $\mathrm{CC} 3.0$ & Loss & Xp22.33 & $0.37-0.78$ & 1.6 & Telomere & $780 \mathrm{~Kb}$ & $\begin{array}{l}\text { SHOX, PLCXD1, GTPBP6, } \\
\quad P P P 2 R 3 B, C S F 2 R A\end{array}$ \\
\hline 11 & $\mathrm{SGC}$ & Gain $^{a}$ & $7 q 11.23$ & 74.91 & 73.6 & 75.27 & $175.8 \mathrm{~Kb}$ & $H I P 1$ \\
\hline 12 & $\mathrm{SGC}$ & Gain $^{a}$ & $7 \mathrm{q} 36.3$ & 158.65 & 158.33 & Telomere & $4.36 \mathrm{~Kb}$ & $V I P R 2$ \\
\hline 13 & $\mathrm{SGC}$ & Gain $^{a}$ & $15 q 11$ & $20.40-20.45$ & Centromere & 20.61 & $50 \mathrm{~Kb}$ & CYFIP1 \\
\hline 14 & $\mathrm{CC} 2.0$ & Gain $^{a}$ & $17 \mathrm{q} 25$ & $69.88-70.08$ & 68.84 & 70.37 & $200 \mathrm{~Kb}$ & DNAI2, SLC9A3R1 \\
\hline
\end{tabular}

Genes listed in last column represent disease genes and other select genes located in region of gain or loss.

${ }^{a}$ Initially reported as abnormal and subsequently determined to be polymorphic variant.

$\mathrm{CC}$, Bluegnome Cytochip (and version number); SGC, Spectral Genomics Constitutional; Mb, megabase; Kb, kilobase; PFC, proximal (relative to centromere) flanking clone position; DFC, distal (relative to centromere) flanking clone position; N/A, not applicable.

15q11) was in a region known to contain heritable duplications, which have been thought to be polymorphic in the human population. Later all four gains were reclassified as probable copy number variants due to their appearing in the Database of Genomic Variants (http://projects.tcag.ca/variation/). We compared the results for our adult population with pediatric samples obtained during the same time frame and analyzed under the same platforms by the same laboratory. We used identical exclusion criteria, i.e., prior abnormal karyotype, no karyotype before array analysis and/or results considered to be copy number variants. The overall abnormality rate in our sampled pediatric population is $11 \%$, which is very similar to prior published reports.

\section{DISCUSSION}

The introduction of clinical aCGH has transformed the approach to intellectual disability in the clinical genetics field and in a span of less than 5 years aCGH has moved from an "emerging technology" to the likely "test of choice" in idiopathic cases of intellectual disability. ${ }^{5,14}$ The majority of published data are derived from studies of entirely or predominantly pediatric-based populations and whether similar abnormalities are found in adult populations had not been well studied. In this project, we provide the first data on an adult population of consecutively evaluated patients that showed aCGH-detected genomic losses in $22 \%$ of our patients. Even when the three patients with detectable high-resolution chromosome abnormalities are removed from the analysis, the prevalence of $17 \%$ of positive aCGH findings is still higher than expected from the literature. ${ }^{11}$ Our data demonstrate that aCGH genomic losses may be relatively commonly found in adults with intellectual disability and that such chromosomal defects are quite compatible with survival to adulthood. The findings have obvious relevance to the genetic evaluation of this population and to genetic counseling approaches to adult patients with intellectual disability. Although the majority of pathogenic gains and losses are usually presumed to be de novo events, our findings suggest that reproductive counseling for adults with unexplained intellectual disabilities needs to account for the possibility of detectable genomic abnormalities that could be transmitted to offspring. Providing genetic counseling for these results raises challenges driven in part by the level of intellectual disability of the patient and the concomitant needs of cognitively normal biological relatives who may also have a vested interest in knowledge of the results. For three of our patients with abnormal results, members of their biological family were directly involved in the care of the intellectually disabled adult. Although the "affected" patients in these instances were unlikely to have children of their own, the risk assessment information was perceived to be of value to their relative. Beyond the reproductive risk assessment discussions, the counseling ses- 
Table 3 Summary of clinical features in adults with aCGH deletions

\begin{tabular}{|c|c|c|c|c|c|}
\hline $\begin{array}{l}\text { Patient } \\
\text { number }\end{array}$ & $\begin{array}{l}\text { Age } \\
\text { (yr) }\end{array}$ & Gender & $\begin{array}{c}\text { Intellectual } \\
\text { disability }\end{array}$ & Clinical features & Family history \\
\hline 1 & 28 & Female & $\begin{array}{l}\text { Severe to } \\
\text { profound }\end{array}$ & $\begin{array}{l}\text { Bitemporal narrowing, low set, posteriorly rotated ears, } \\
\text { proptosis, strabismus, short nose with bulbous tip, flat } \\
\text { philtrum, thin upper lip, small hands, low muscle } \\
\text { tone, scoliosis, and hearing loss }\end{array}$ & $\begin{array}{l}\text { Distant relative with profound } \\
\text { ID; multiple individuals } \\
\text { with clubfoot }\end{array}$ \\
\hline
\end{tabular}

$2 \quad 42 \quad$ Male $\quad \begin{gathered}\text { Severe to } \\ \text { profound }\end{gathered}$

Nonverbal, nonambulatory, prematurely gray hair, history of white forelock, prominent jaw, deep set eyes, contractures, tapered fingers, hearing loss, and previous seizure activity

Myopathic facies, mild strabismus, mild ptosis, full lips, high arched palate, bilateral transverse palmar creases, tapered fingers, decreased muscle tone, seizures, foodseeking behavior

Mild

Posteriorly rotated, small, low-set ears, downslanting palpebral fissures, broad nasal root, high arched palate, lordosis, bilateral proximally placed 5 th finger, bilateral deviated great toes, bilateral pes planus, $\mathrm{mitral} /$ tricuspid valve regurgitation, depression

Macrocephaly, coarse features, mild synophorys, deep set eyes, prominent supraorbital ridges, bulbous nasal tip, thick lips, hydrocephalus, agenesis of corpus callosum, contractures

right low set ear with simple folds, deep set eyes, horizontal eyebrows, prominent forehead, flat palate, small mouth, thin upper lip, prominent jaw, tapered fingers, shuffling gait, psychiatric disorder

\begin{tabular}{|c|c|c|c|}
\hline 7 & 44 & Male & Mild \\
\hline 8 & 38 & Female & Mild \\
\hline 9 & 43 & Male & Moderate \\
\hline 0 & 21 & Female & Moderate \\
\hline 1 & 27 & Female & Mild \\
\hline 12 & 30 & Male & Moderate \\
\hline 13 & 34 & Male & Mild \\
\hline 14 & 52 & Female & Mild \\
\hline
\end{tabular}

Macrocephaly, obese, prominent forehead, bilateral large ears, deep-set eyes, downslanting palpebral fissures, myopathic facies, high arched palate, left single palmar crease, history of seizures

Microcephaly, elongated facies, bitemporal wasting, deep set eyes, hypotelorism, narrow jaw and palate, full lips, short stature, small hands, 5th finger clinodactyly, shortened 5th fingers, broad proximally-placed thumbs, bilateral pes planus, abnormal gait, significant speech/language delays, late-onset hearing loss

Sparse hair, hand atrophy, shuffling gait, high-pitched voice, history of pyloric stenosis, history of seizures, enlarged ventricles, cerebral volume loss, and skull thickening on brain imaging, cognitive decline, psychiatric disorder

Short stature, Madelung deformity, tibial shortening, nondysmorphic facies, thick neck, abdominal obesity, bilateral brachydactyly, psychiatric disorder

Short stature, high arched palate, minimal hyperpigmentation, psychiatric disorder

Broad palate, tremors, seizure disorder, poor weight gain, osteoporosis, behavioral problems

Short stature, thin upper lip, broad eyebrows, protuberant lower lip, right simian crease, small hands, broad thumbs, broad toes, bilateral shortened 4 and 5 digits, hypertension, type 1 diabetes

Cushingoid appearance, retrognanthia, small mouth, crowded teeth, small underdeveloped ears, antihelices, short neck, short stature, cleft palate, tricuspid/pulmonary atresia, renal artery stenosis, seizures, transient ischemic attack

Unknown

Negative

Half-brother with ID

negative

brother with ID

Unknown

Negative

Negative

Unknown

Negative

Identical twin brother with mild ID

Negative

Nephew with CHD, paternal first cousin with ID 
sions also brought out a general sense of satisfaction from family members that a biological explanation was now evident for the cause of their relative's intellectual disability.

Continued improvements in pediatric medical and social care will continue to grow in the population of adults with intellectual disability. Our experience is that few of these patients have been evaluated by geneticists or genetic counselors and that "modern" molecular genetic testing has rarely been applied, and it may be necessary to develop educational programs to encourage providers and stakeholders to refer such patients for genetic evaluations that include aCGH. The message that a prior "negative genetic workup" done 20 or more years ago is not sufficient to have excluded genetic causes of intellectual disability needs to be conveyed to families and primary care physicians. The potential to identify causative genomic abnormalities is important for family risk counseling, especially to adult-siblings who are often in their own reproductive years and could receive accurate recurrence risk assessment in cases where an aCGH abnormality is detected in their affected sibling. Of greater importance, perhaps, is the potential recurrence risk for affected patients with genomic defects, some of whom are involved in interpersonal relationships and may want children of their own. Presumably, the recurrence risk estimates for offspring of affected patients with genomic abnormalities are substantially greater than the recurrence risks for parents of an affected child who desire additional children where the event in their affected child is most likely de novo. Unfortunately, recurrence risk data for offspring of persons with such genomic defects is largely lacking as are validated genetic counseling approaches to explain known and theoretical reproductive risks when one or both members of a couple has intellectual deficits.

Although yields of cytogenetically visible chromosomal anomalies are higher with increasing severity of intellectual phenotype, it is important to note that we detected genomic losses in mild and moderate disease. Larger studies are needed to determine whether yields of aCGH differ based on severity of cognitive deficit in adult patients as it may in pediatric cases. As suggested above, the presence of genomic losses in mild cases, the largest and most reproductively capable subgroup, have important implications for delivery of genetic counseling to patients and families. With the prevalence of defects in the range of $20 \%$ in our study, it is likely that some couples where both prospective parents have mild to moderate intellectual disability may both harbor genomic abnormalities, further complicating the genetic counseling approach.

It is possible that the lower rates of aCGH findings in some pediatric studies reflect the fact that a proportion of pediatric referrals for intellectual disability will prove in retrospect to be cases of temporary developmental delay that ultimately resolve or can be improved with therapy. Thus, the population of pediatric patients that undergo aCGH testing is probably broader than the adult population and includes a proportion of children who will ultimately be classified as having normal cognition and will also presumably have a lower rate of abnormal aCGH findings. If this is true, then abnormal aCGH findings may prove to be a marker for cognitive problems that are more likely to persist into adulthood. Patients with aCGH genomic findings showed multiple, mild dysmorphic features on evaluation by our geneticist. However, in most cases, the dysmorphic findings were relatively mild, and no clear statistically significant difference between patients with normal or abnormal aCGH studies was noted. Making clear correlations between genomic defects and phenotype characteristics are difficult in our study because each finding was unique in our population. Although no exact matches for our findings were noted in public databases, some overlaps were observed. For instance, a $9.68 \mathrm{Mb}$ deletion on chromosome X (Patient 872, DECIPHER: http://www.decipher.sanger.ac.uk) reportedly had obesity, short stature, and brachydactyly, similar findings to our patient deleted in this region. We also noted a reported patient with retinal dysplasia, autism, and microcephaly (Patient 595, DECIPHER) with a duplication of $5 \mathrm{q} 13.3 \mathrm{q} 15(17.57 \mathrm{Mb})$ of a similar region to the one deleted in our patient with agenesis of the corpus callosum, profound mental retardation, and macrocephaly. However, at the present time, it is unclear whether such apparent similarities or patterns between genomic gains or losses are clinically meaningful.

Our study was limited by the modest size of our study population, and although we included consecutively evaluated patients, we were unable to control for any referral bias that influenced which patients were referred by physicians to our clinic. Larger studies are needed to determine whether the relatively high rate of aCGH findings in our adult cohort can be replicated in other populations. Collection of detailed clinical phenotype data remains an important component of these studies to best correlate genomic abnormalities with clinical outcomes including the delineation of which genomic losses and gains are compatible with survival into adulthood. For example, several groups have now reported on unrelated pediatric cases of 5q14.3-q15 genomic losses detected using aCGH or single nucleotide polymorphism arrays. ${ }^{15-17}$ Patients were identified in infancy or early childhood, with the oldest reported patient being 7 years old. The common overlapping phenotypes include severe intellectual disability, central nervous system abnormalities, hypotonia, and seizures. Two genes in this region GPR98 and $M E F C 2$ have been proposed as candidates to explain the phenotypes seen including the seizures, which are felt to be due to GPR98, previously identified as a "seizure" gene. The structural brain anomalies could be due to the loss of $M E F C 2$, which is proposed to influence neuronal migration. Our patient, at 37 years is the oldest $15 \mathrm{q} 14.3$-q15 patient reported to our knowledge. Despite profound intellectual disability and requirements for round-the-clock care, she has had few hospitalizations and does not have any chronic progressive medical illnesses. In distinction to the majority of the pediatric cases, she does not suffer from seizures and we estimate her chances of long-term survival to be quite high, and her case provides important prognostic information for the families with young children with this recurrent microdeletion condition.

We also used different platforms with increasing resolution throughout the study. As these tests were done clinically, we have not gone back to our earliest cases and retested patients using the newest aCGH available. Thus, we may have underestimated the prevalence of genomic abnormalities by initially using lower-density arrays and by relying on clinical BAC arrays rather than high-density oligonucleotide arrays. ${ }^{18}$ During the course of the study, we did detect four genomic gains, which were initially interpreted as having pathogenic potential. Maturation of the published data on benign genomic variation ultimately proved these changes to be polymorphic. That these variants were reclassified by us clinically in a relatively short period of time speaks to the fact that this technology is relatively still new and results must be interpreted carefully. Obtaining parental samples as a method to determine whether an aCGH finding is de novo can be extremely difficult in the adult intellectually disabled population because of the frequent unavailability of relatives due to severing of family ties, unknown whereabouts, and/or death of biological relatives. 


\section{ACKNOWLEDGMENTS}

Supported by March of Dimes Foundation.

\section{REFERENCES}

1. Fujiura GT. Continuum of intellectual disability: demographic evidence for the "forgotten generation". Ment Retard 2003;41:420-429.

2. McLaren J, Bryson SE. Review of recent epidemiological studies of mental retardation: prevalence, associated disorders, and etiology. Am J Ment Retard 1987;92:243-254.

3. Roeleveld N, Zielhuis GA, Gabreels F. The prevalence of mental retardation: a critical review of recent literature. Dev Med Child Neurol 1997;39:125132 .

4. Moeschler JB. Genetic evaluation of intellectual disabilities. Semin Pediatr Neurol 2008;15:2-9.

5. Moeschler JB, Shevell M. Clinical genetic evaluation of the child with mental retardation or developmental delays. Pediatrics 2006;117:23042316.

6. van Karnebeek CD, Jansweijer MC, Leenders AG, Offringa M, Hennekam RC. Diagnostic investigations in individuals with mental retardation: a systematic literature review of their usefulness. Eur J Hum Genet 2005;13: $6-25$.

7. Beange H, McElduff A, Baker W. Medical disorders of adults with mental retardation: a population study. Am J Ment Retard 1995;99:595-604.

8. Fisher K, Kettl P. Aging with mental retardation: increasing population of older adults with MR require health interventions and prevention strategies. Geriatrics 2005;60:26-29.

9. Honeycutt AA, Grosse SD, Dunlap LJ, et al. Economic costs of mental retardation, cerebral palsy, hearing loss, and vision impairment, in using survey data to study disability: results from the National Health Interview Survey on disability. Research in social science and disability. Amsterdam, Holland: Elsevier, 2003.
10. Prater CD, Zylstra RG. Medical care of adults with mental retardation. Am Fam Physician 2006;73:2175-2183

11. Sagoo GS, Butterworth AS, Sanderson S, Shaw-Smith C, Higgins JP, Burton $\mathrm{H}$. Array $\mathrm{CGH}$ in patients with learning disability (mental retardation) and congenital anomalies: updated systematic review and meta-analysis of 19 studies and 13,926 subjects. Genet Med 2009;11:139-146.

12. Shao L, Shaw CA, Lu XY, et al. Identification of chromosome abnormalities in subtelomeric regions by microarray analysis: a study of 5,380 cases. $\mathrm{Am} \mathrm{J}$ Med Genet A 2008;146A:2242-2251.

13. Lu X, Shaw CA, Patel A, et al. Clinical implementation of chromosomal microarray analysis: summary of 2513 postnatal cases. PLoS One 2007;2: e327.

14. Edelmann L, Hirschhorn K. Clinical utility of array CGH for the detection of chromosomal imbalances associated with mental retardation and multiple congenital anomalies. Ann N Y Acad Sci 2009;1151:157-166.

15. Cardoso C, Boys A, Parrini E, et al. Periventricular heterotopia, mental retardation, and epilepsy associated with 5q14.3-q15 deletion. Neurology 2009;72:784-792.

16. Engels H, Wohlleber E, Zink A, et al. A novel microdeletion syndrome involving 5q14.3-q15: clinical and molecular cytogenetic characterization of three patients. Eur J Hum Genet. 2009;17:1592-1599.

17. Le Meur N, Holder-Espinasse M, Jaillard S, et al. MEF2C haploinsufficiency caused either by microdeletion of the $5 \mathrm{q} 14.3$ region or mutation is responsible for severe mental retardation with stereotypic movements, epilepsy and/or cerebral malformations [published online ahead of print July 9 , 2009]. J Med Genet doi: 10.1136/jmg.2009.069732.

18. Aradhya S, Manning MA, Splendore A, Cherry AM. Whole-genome arrayCGH identifies novel contiguous gene deletions and duplications associated with developmental delay, mental retardation, and dysmorphic features. Am J Med Genet A 2007;143A:1431-1441.

19. Shaffer LG, Tommerup N (Eds.). ISCN 2005: an international system for human cytogenetic nomenclature. Basel, Switzerland: S. Karger, 2005. 